\title{
UN NOUVEAU CAS DE CONVERGENCE DES SÉRIES DE FOURIER.
}

\author{
Par M. Ch.-J. de la Vallée Poussin (Louvain).
}

Adunanza del I2 febbrajo I9II.

r. Soit $f(x)$ une fonction sommable (au sens de M. Lebesgue) dans l'intervalle $(\mathrm{o}, 2 \pi) ; x$ un point de cet intervalle. La somme $S_{n}$ des $n+$ I premiers termes de la série de Fourier de $f(x)$ a pour expression connue

$$
S_{n}=\frac{\mathrm{I}}{\pi} \int_{-\frac{x}{2}}^{\pi-\frac{x}{2}} f(x+2 u) \frac{\sin (2 n+\mathrm{I}) u}{\sin u} d u .
$$

Soit $\varepsilon$ un nombre positif donné d'une petitesse arbitraire. Quand $n$ croit indéfiniment, la valeur asymptotique de $S_{n}$ est, d'après un théorème connu de M. LebesGue '), la même que celle de

ou encore que celle de

$$
\frac{\mathrm{I}}{\pi} \int_{-\varepsilon}^{+\varepsilon} f(x+2 u) \frac{\sin (2 n+\mathrm{I}) u}{\sin u} d u
$$

$$
\frac{\mathrm{I}}{\pi} \int_{-\varepsilon}^{+\varepsilon} f(x+2 u) \frac{\sin (2 n+\mathrm{I}) u}{u} d u
$$

car la différence de ces deux intégrales est de la forme $\int \varphi(u) \sin (2 n+\mathrm{I}) u d u$, où q est sommable et cette intégrale tend vers zéro pour $n=\infty$, en vertu du théorème déjà invoqué de M. Lebesgue. En définitive, la valeur asymptotique de $S_{n}$ est la même que celle de la dernière intégrale, qui s'ècrit encore

$$
\frac{\mathrm{I}}{\pi} \int_{0}^{\varepsilon}[f(x+2 u)+f(x-2 u)] \frac{\sin (2 n+\mathrm{I}) u}{u} d u .
$$

Pour prouver la convergence et trouver la somme de la série de Fourier, il suffit donc de prouver l'existence et de trouver la limite pour $k=\infty$ de l'expression

$$
V=\frac{\mathrm{I}}{\pi} \int_{0}^{\varepsilon}[f(x+2 u)+f(x-2 u)] \frac{\sin k u}{u} d u .
$$

1) Leçons sur les séries trigonométriques (Paris, Gauthier-Villars, 1906). 
2. Posons

$$
F(u)=\frac{\mathrm{I}}{2 u} \int_{0}^{u}[f(x+2 u)+f(x-2 u)] d u .
$$

Il vient, par une intégration par parties, effectuée sur $f(x+2 u)+f(x-2 u)$,

$$
\frac{\pi}{2} V=F(\varepsilon) \sin k \varepsilon-\int_{0}^{\varepsilon} F(u) k \cos k u d u+\int_{0}^{\varepsilon} F(u) \frac{\sin k u}{u} d u .
$$

Voici maintenant l'hypothèse qui va nous conduire à une nouvelle règle de convergence, ce sera que $F(u)$ est à variation bornée dans l'intervalle $(o, \varepsilon)$.

On a, dans ce cas, par un théorème classique,

$$
\lim _{k=\infty} \int_{0}^{\varepsilon} F(u) \frac{\sin k u}{u} d u=\frac{\pi}{2} F(+0) .
$$

Il suffit donc pour établir que $V$, donc que la série de Fourier, converge vers $F(+o)$ au point $x$, de prouver que l'ensemble des deux termes précédents:

$$
F(\varepsilon) \sin k \varepsilon-\int_{0}^{\varepsilon} F(u) k \cos k u d u
$$

est aussi petit que l'on veut avec $\varepsilon$.

Or cette expression est la différence des deux quantités:

$$
[F(\varepsilon)-F(+0)] \sin k \varepsilon, \quad \int_{0}^{\varepsilon}[F(u)-F(+o)] k \cos k u d u .
$$

Il suffit donc de prouver que chacune d'elles est aussi petite que l'on veut avec $\varepsilon$.

C'est évident pour la première qui est aussi petite que l'on veut avec $F(\varepsilon)-F(+0)$. Reste à considérer la seconde.

Soit $T(u)$ la variation totale de $F(u)$ dans l'intervalle $(o, u)$. Cette fonction étant positive et non décroissante, on a, par le second théorème de la moyenne, $\varepsilon^{\prime}$ étant $<\varepsilon$,

$$
\int_{0}^{\varepsilon} T(u) k \cos k u d u=T(\varepsilon) \int_{\varepsilon^{\prime}}^{\varepsilon} k \cos k u d u .
$$

On a, pour les mêmes raisons, $\varepsilon^{\prime \prime}$ étant aussi $<\varepsilon$,

$$
\int_{0}^{\varepsilon}[T(u)-F(u)+F(+o)] k \cos k u d u=[T(\varepsilon)-F(\varepsilon)+F(+o)] \int_{\varepsilon^{\prime \prime}}^{\varepsilon} k \cos k u d u \text {. }
$$

La quantité qui nous occupe est la différence des deux intégrales que nous venons de transformer. Elle est donc bien aussi petite que l'on veut avec $\varepsilon$, car ces deux intégrales sont aussi petites que l'on veut avec $T(\varepsilon)$ et $F(\varepsilon)-F(+o)$, puisque $\int k \cos k u d u$ est de valeur absolue moindre que 2 .

On peut donc formuler le théorème suivant:

3. ThÉorème. - Si la fonction $f(x)$ est sommable dans l'intervalle $(0,2 \pi)$ et que 
la fonction ${ }^{2}$ )

$$
F(u)=\frac{\mathrm{x}}{2 u} \int_{0}^{u}[f(x+u)+f(x-u)] d u
$$

soit à variation bornée quand $u$ tend vers zéro, la série de Fourier de $f(x)$ converge vers $F(+0)$ au point $x$ compris dans l'intervalle $(0,2 \pi)$.

4. Le criterium précédent renferme comme cas particulier celui de $f(x)$ d variation bornée, car, si une fonction est croissante, sa valeur moyenne l'est aussi.

Nous allons montrer que, $f(x)$ étant supposée continue dans le voisinage du point $x$, ce criterium renferme aussi comme cas particulier celui qui est relatif a l'existence de l'intégrale

où l'on a posé, en abrégé,

$$
\int_{0}^{u}|\varphi(u)| \frac{d u}{u}
$$

$$
\varphi(u)=f(x+u)+f(x-u)-2 f(x) .
$$

Il faut, en effet, montrer pour cela que l'existence de cette intégrale entraine celle de

$$
\int_{0}^{\epsilon}\left|F^{\prime}(u)\right| d u \text {. }
$$

Or $F^{\prime}(u)$ a pour valeur (dans le voisinage de $u=0$ )

$$
\frac{f(x+u)+f(x-u)}{2 u}-\frac{1}{2 u^{2}} \int_{0}^{u}[f(x+u)+f(x-u)] d u,
$$

ou, ce qui est la même chose,

Par conséquent, on a

$$
\frac{\varphi(u)}{2 u}-\frac{\mathrm{I}}{2 u^{2}} \int_{0}^{u} \varphi(u) d u
$$

$$
\int_{0}^{\varepsilon}\left|F^{\prime}\right| d u<\int_{0}^{\varepsilon}\left|\frac{\varphi(u)}{2 u}\right| d u+\int_{0}^{\varepsilon} \frac{d u}{2 u^{2}} \int_{0}^{u}|\varphi(u)| d u .
$$

Par le changement de la variable d'intégration $u$ en $u t$, l'inversion des intégrations, puis un nouveau changement de $u$ en $u: t$, cette dernière intégrale double devient successivement ${ }^{3}$ ):

$$
\int_{0}^{\varepsilon} \frac{d u}{2 u} \int_{0}^{1}|\varphi(u t)| d t=\int_{0}^{1} \frac{d t}{2} \int_{0}^{\varepsilon}|\varphi(u t)| \frac{d u}{u}=\int_{0}^{1} \frac{d t}{2} \int_{0}^{e t}|\varphi(u)| \frac{d u}{u} .
$$

Elle est donc moindre que

Il vient donc, en définitive,

$$
\frac{\mathrm{I}}{2} \int_{0}^{\varepsilon}|\varphi(u)| \frac{d u}{u}
$$

$$
\int_{0}^{\varepsilon}\left|F^{\prime}\right| d u<\int_{0}^{\varepsilon}\left|\frac{p(u)}{u}\right| d u
$$

ce qui prouve la proposition.

2) On écrirait $F\left(\frac{u}{2}\right)$ dans la notation précédente.

3) Ces transformations ne soulèvent d'ailleurs aucune objection. 
Dans le raisonnement précédent, nous avons supposé, pour plus de facilité, que la fonction $f(x)$ était continue dans le voisinage du point $x$. Il y a lieu d'observer que la continuité au seul point $x$ suffit. En effet, l'expression trouvée pour $F^{\prime}$ reste valable sauf dans un ensemble de points de mesure nulle, il reste donc légitime de l'introduire dans les int'grales, et les conclusions subsistent.

Louvain, le 8 février $191 \mathrm{I}$.

Ch. - J. De la Vallée Poussin. 